\title{
Benefits and risks of induction of labor at 39 or more weeks in uncomplicated nulliparous women: a retrospective, observational study
}

\author{
Hye In Kim, Sung Pil Choo, Sang Won Han, Eui Hyeok Kim \\ Department of Obstetrics and Gynecology, National Health Insurance Service Ilsan Hospital, Goyang, Korea
}

\section{Objective}

To critically compare the benefits and risks of labor induction versus spontaneous labor in uncomplicated nulliparous women at 39 or more weeks of gestation.

\section{Methods}

We conducted a retrospective, observational study of 237 nulliparous women who were at 39 or more weeks of a singleton pregnancy with vertex presentation and intact membranes. We compared maternal outcomes including the Cesarean section rate and neonatal outcomes in the induced labor and spontaneous labor groups.

Results

Among the 237 women, 199 delivered vaginally (84.0\%). The spontaneous labor group and induced labor group had a similar incidence of Cesarean delivery (17.7\% vs. $12.3 \%, P=0.300)$. The length of stay and blood loss during delivery were also similar between the groups ( $4.3 \pm 1.5$ vs. $3.9 \pm 1.5$ days and $1.9 \pm 1.3$ vs. $1.8 \pm 1.0 \mathrm{mg} / \mathrm{sL}$, respectively; all $P>0.05)$. Regarding neonatal outcomes, the rate of meconium-stained amniotic fluid, Apgar score $<7$ at 5 minutes, and intubation rate were similar between the groups ( $18.9 \%$ vs. $24.7 \%, 7.9 \%$ vs. $4.1 \%$, and $6.1 \%$ vs. $4.4 \%$, respectively, all $P>0.05)$. Only the neonatal intensive care unit admission rate was significantly lower in the induction group than in the spontaneous labor group $(28.0 \%$ vs. $13.2 \%, P=0.001)$.

\section{Conclusion}

Maternal adverse outcomes of labor induction at 39 weeks of gestation were similar to those in a spontaneous labor group in uncomplicated nulliparous women. Neonatal adverse events were also similar between the groups. It may be acceptable to schedule labor induction as long as 7 days before the estimated date, even when the indication is only relative.

Keywords: Induced labor; Cesarean section; Newborn infant; Postpartum period; Obstetric delivery

\section{Introduction}

Induced labor represents the most frequent interventional procedure in the field of obstetric medicine; it is reportedly applied in $20 \%$ to $25 \%$ of all pregnancies [1,2]. Induced labor is indicated in situations in which the outcomes for the mother and neonate are better if the pregnancy is not further prolonged $[2,3]$.

There are potential medical advantages to scheduled induction of labor at full term, such as reduction in stillbirth and further fetal growth, which leads to macrosomia and its consequences [4-6]; moreover, elective labor induction can
Received: 2018.03.05. Revised: 2018.07.15. Accepted: 2018.07.31. Corresponding author: Eui Hyeok Kim

Department of Obstetrics and Gynecology, National Health Insurance Service Ilsan Hospital, 100 Ilsan-ro, Ilsandong-gu, Goyang 10444, Korea

E-mail: raksumi10@gmail.com https://orcid.org/0000-0002-3264-3620

Articles published in Obstet Gynecol Sci are open-access, distributed under the terms of the Creative Commons Attribution Non-Commercial License (http://creativecommons. org/licenses/by-nc/3.0/) which permits unrestricted non-commercial use, distribution, and reproduction in any medium, provided the original work is properly cited.

Copyright $\odot 2019$ Korean Society of Obstetrics and Gynecology 


\section{Obstetrics \& Gynecology Science}

Vol. 62, No. 1, 2019

reduce the chance of sudden disruption of the patient's life and provider's work. In addition, regarding neonatal outcomes, studies have shown that an increased risk for perinatal and maternal complications was detected as early as 40 weeks of gestation [7].

However, elective induction may be associated with drawbacks such as increased length of labor, the potential for patient/provider impatience, Cesarean delivery, a long latent phase, increased cost, and neonatal morbidity if the gestational age is less than 39 weeks of gestation $[3,8]$. The Cesarean delivery rate, which is thought to be the most important and adverse outcome of labor induction, is inversely correlated with cervical favorability at induction, based on the Bishop score $[9,10]$; thus, the cervical status may represent one of the most important predictors of successful vaginal delivery when deciding to induce labor.

Some studies, however, have indicated there is no convincing evidence that elective labor induction is associated with a substantial increase in the rate of Cesarean delivery when the rate at term in electively induced women is compared to that of women at the same gestational age managed expectantly [5,11-14].

Furthermore, Saccone and Berghella [15] recently demonstrated that the rate of Cesarean delivery was not increased by induction at full term, even for mothers with an unfavorable cervix, and Miller et al. [16] reproduced this result by conducting a randomized, controlled trial in 2015.

The American College of Obstetricians and Gynecologists (ACOG) has stated that induced labor between 37 and 38 gestational weeks, i.e., early term, should be avoided in uncomplicated women [17]. This is because early term newborns have greater neonatal morbidity and healthcare utilization during their entire first year of life than those born at 39 to 40 weeks of gestation [18-20].

A recent study showed that the overall frequency of labor induction more than doubled in the United States, increasing from $9.5 \%$ in 1990 to $23.8 \%$ in 2010 [21], and elective induction for the mother and provider's convenience accounted for approximately $40 \%$ of cases [22]. Some authors supported induced labor at 39 weeks or more $[12,23]$. If elective induction is considered at term, inherent risks must be discussed, informed consent must be obtained, and guidelines must be followed, as promulgated by the ACOG [24].

The purposes of this study were to critically compare the benefits and risks of labor induction versus spontaneous labor in uncomplicated singleton gestations at 39 or more weeks of gestation and to evaluate whether induction of labor at full term in low-risk women reduces the risk of composite maternal and perinatal morbidity.

\section{Materials and methods}

This study was an observational, retrospective study conducted between January 1, 2011 and November 30, 2017 at the National Health Insurance Service Ilsan Hospital in the Republic of Korea.

All women from 39 gestational weeks, 0 days to 41 gestational weeks, 6 days were considered for participation in this study. When women were at 39 gestational weeks or more in the outpatient department of the hospital (OPD), they were given the choice between waiting for spontaneous labor or inducing labor. The following inclusion criteria were used: 1) primiparous women, 2) uncomplicated living singleton pregnancy, 3) gestational age from 39 weeks, 0 days to 41 weeks, 6 days, 4) cephalic presentation, and 5) intact amniotic membrane. Women undergoing induced labor due to premature rupture of membranes, which can alter the course of labor, and women with indications for Cesarean delivery, such as placenta previa, previous Cesarean section status, or previous myomectomy were excluded. We excluded women with a myoma more than $8 \mathrm{~cm}$, those with uncontrollable diabetes, or those with other severe medical diseases, such as poorly controlled gestational hypertension. Additionally, we excluded women with fetuses with intrauterine growth restriction or large-for-gestational-age fetuses.

Women in the induction group were admitted to the delivery room via the OPD. Near term, most women have Braxton-Hicks contractions, which are not painful and discovered incidentally on a non-stress test. Because Braxton-Hicks contractions are not accompanied by change in the cervix length, we did not consider them as spontaneous labor. Most women in the spontaneous labor group were admitted directly to the delivery room, or some came to the OPD with complaints of pain and regular labor. All patients were clinically evaluated by pelvic examination to determine the Bishop scores for cervical dilatation, effacement, consistency, and the position and station of the fetus [10]. These $5 \mathrm{com}$ ponents of the Bishop score were measured by 1 expert (EHK). Fetal head engagement in the maternal pelvic cavity was also 


\section{Obstetrics \& Gynecology Science}

Hye In Kim, et al. Induction, labor, risks

assessed by pelvic examination at this time.

Next, ultrasonography was performed by 1 expert (EHK) using the Philips Ultrasound IU22 (Bothell, WA, USA) and EPIQ 7 (Bothell, WA, USA) with a vaginal probe. The probe was inserted vaginally $3 \mathrm{~cm}$ from the cervix, and the length between the internal and external os of the cervix in the longitudinal section was measured in accordance with previously validated technical criteria [25]. The cervical longitudinal section was defined by the view of the cervical canal, and the cervical length was defined as the shortest value based on 4 or more measurements.

Labor induction was attempted with oxytocin (intravenous injection, $10 \mathrm{lU} / \mathrm{mL}$; Pitocin, Jeil Pharmaceutical Co. Ltd., Daegu, Korea) or prostaglandin E2 (intravaginally, $10 \mathrm{mg}$; Propess, Bukwang Pharm Co. Ltd., Seoul, Korea), and when women developed spontaneous labor pain, augmentation with oxytocin was attempted if labor progression was inadequate. Fetal heart rate was continuously monitored by cardiotocography between 30 minutes before and 1 hour after oxytocin or prostaglandin E2 administration in all women. Prostaglandin E2 was inserted vaginally and removed 12 hours after the insertion or earlier in case of onset of active labor, rupture of membranes, or abnormal cardiotocography findings (i.e., fetal hyperstimulation or other alteration in the fetal heart rate). If non-reassuring fetal heart pattern developed, we discontinued oxytocin temporarily. In the spontaneous labor group, we used oxytocin when we thought labor progression was inadequate.
From the institution's electronic medical record, we obtained the following information: data concerning the delivery mode (vaginal or Cesarean delivery), time between admission and vaginal delivery, duration of the second stage, maternal age, gestational age, change in the hemoglobin level after delivery (as a decrease implies blood loss during the delivery), and length of hospital stay. To evaluate neonatal complications, we assessed the neonatal weight, 1- and 5-minute Apgar scores, neonatal intensive care unit (NICU) admission rate, meconium status, and neonatal intubation status.

\section{Statistical analyses}

Demographic and clinical characteristics were compared between women with and without induced labor using Student's $t$-test for continuous values and the $\chi^{2}$ test or Fisher's exact test for categorical values. We obtained odds ratios for successful vaginal delivery using a logistic regression model. All $P$-values were 2 -tailed, and $P<0.05$ was considered statistically significant. All analyses were performed using the Statistical Package for Social Sciences, version 23.0 (SPSS Inc., Chicago, IL, USA).

\section{Results}

A total of 237 women were recruited for this study; there were 73 women in the expectant group and 164 women in

Table 1. Characteristics of women and Comparison of characteristics according to Induced labor $(n=237)$

\begin{tabular}{lcccc}
\hline \multirow{2}{*}{ Characteristics } & \multirow{2}{*}{ Total } & \multicolumn{2}{c}{ Labor } & \multirow{2}{*}{$\boldsymbol{P}$-value } \\
\cline { 3 - 4 } & & Spontaneous (n=73) & Induced (n=164) & \\
\hline Age (yr) & $31(19-42)$ & $31(20-42)$ & $31(19-41)$ & 0.958 \\
Gestational age $(\mathrm{wk})$ & $39(39-41)$ & $39(39-41)$ & $39(39-41)$ & 0.442 \\
Pre-pregnancy BMl $\left(\mathrm{kg} / \mathrm{m}^{2}\right)$ & $21.8 \pm 3.6$ & $21.5 \pm 2.7$ & $21.9 \pm 3.9$ & 0.550 \\
Bishop score & $4(0-10)$ & $5(0-10)$ & $4(0-8)$ & $<0.001^{\text {b) }}$ \\
Cervical length (mm) & $17.4 \pm 8.1$ & $14.8 \pm 6.8$ & $18.5 \pm 8.3$ & $<0.001^{\text {b) }}$ \\
Cervical funneling & $111(46.8)$ & $36(51.4)$ & $75(45.7)$ & 0.424 \\
Engagement of fetal head & $205(86.5)$ & $67(91.8)$ & $138(84.1)$ & 0.112 \\
Neonatal birthweight (g) & $3,242 \pm 420$ & $3,273 \pm 406$ & $3,228 \pm 426$ & 0.449 \\
Neonatal birthweight greater than 3,500 g & $65(27.4)$ & $18(24.7)$ & $47(28.7)$ & 0.524 \\
\hline
\end{tabular}

Values are presented as the median (range), mean \pm standard deviation, or number (\%).

BMI, body mass index.

${ }^{\text {a) }}$ Total possible score $=13 ;{ }^{\text {b) }}$ Statistical significance. 


\title{
Obstetrics \& Gynecology Science
}

\author{
Vol. 62, No. 1, 2019
}

Table 2. Comparison of maternal/neonatal outcomes according to Induced labor

\begin{tabular}{|c|c|c|c|}
\hline \multirow{2}{*}{ Variables } & \multicolumn{2}{|c|}{ Labor } & \multirow{2}{*}{$P$-value } \\
\hline & Spontaneous $(n=73)$ & Induced $(n=164)$ & \\
\hline Cesarean section rate & $9(12.3)$ & $29(17.7)$ & 0.300 \\
\hline Decrease in Hgb after delivery (g/dL) & $1.8 \pm 1.0$ & $1.9 \pm 1.3$ & 0.606 \\
\hline Time from admission to delivery ${ }^{\text {a) }}$ (min) & $597 \pm 452$ & $924 \pm 549$ & $<0.001^{\text {b) }}$ \\
\hline Delivery within 12 hours ${ }^{\mathrm{a})}$ & $48 / 64(75.0)$ & $68 / 135(50.4)$ & $0.001^{b)}$ \\
\hline Length of Stay (day) & $3.9 \pm 1.5$ & $4.3 \pm 1.5$ & 0.080 \\
\hline AS at $1 \mathrm{~min}$ & $7(2-8)$ & $7(1-9)$ & 0.112 \\
\hline AS at $5 \mathrm{~min}$ & $8(5-9)$ & $8(3-10)$ & 0.176 \\
\hline AS at $5 \min <7$ & $3(4.1)$ & $13(7.9)$ & 0.403 \\
\hline NICU admission & $7(13.2)$ & $46(28.0)$ & $0.001^{b)}$ \\
\hline Meconium-stained amniotic fluid & $18(24.7)$ & $31(18.9)$ & 0.385 \\
\hline Intubation & $3(4.4)$ & $10(6.1)$ & 0.535 \\
\hline
\end{tabular}

Values are presented as the number (\%), mean \pm standard deviation, or median (range).

$\mathrm{Hgb}$, hemoglobin; AS, Apgar score; NICU, neonatal intensive care unit.

a) Included only vaginal delivery; ${ }^{\text {b) }}$ Statistical significance.

the induction group. Among all women, 199 (84.0\%) delivered vaginally.

Table 1 summarizes demographic data and clinical outcomes. The mean gestational age at delivery was 39.4 gestational weeks. Thirty-eight women (16.0\%) required Cesarean delivery.

The mean Bishop score and cervical length before induction were 4.1 and $17.4 \mathrm{~mm}$, respectively, at admission to the delivery room. Among all women, 111 (46.8\%) had cervical funneling, as detected by transvaginal ultrasonography (Table 1).

Between the 2 groups, there was no difference in maternal age, gestational age, pre-pregnancy maternal body mass index, ratio of engagement of the fetal head, neonatal birth weight, and rate of neonates with a birth weight greater than $3,500 \mathrm{~g}$. We set the birth weight cutoff value as 3,500 $\mathrm{g}$ because weight less than $3,500 \mathrm{~g}$ is one of the favorable factors for labor induction $[26,27]$. In contrast, the Bishop score and cervical length were significantly different between the 2 groups (Table 1).

Concerning maternal complications, the Cesarean section rate, decrease in hemoglobin level after delivery, and length of hospital stay were not different between the 2 groups. Delivery time from admission, however, was longer in the induction group than in the spontaneous labor group $(597 \pm 452$ vs. $924 \pm 549$ hours, $P<0.001$ ), and the rate of delivery within 12 hours among women with vaginal delivery was also higher in the spontaneous labor group than in the induction
Table 3. Cervix status according to delivery mode and logistic regression analysis for successful vaginal delivery

\begin{tabular}{|c|c|c|}
\hline Delivery mode & Bishop score & $\begin{array}{c}\text { Cervix length } \\
(\mathrm{mm})\end{array}$ \\
\hline \multicolumn{3}{|c|}{ Spontaneous labor group } \\
\hline $\operatorname{VD}(n=64)$ & $5(1-10)$ & $14.0 \pm 6.5$ \\
\hline$C D(n=9)$ & $3(0-9)$ & $20.6 \pm 8.5$ \\
\hline$P$-value & $<0.001^{\mathrm{a})}$ & $0.008^{\mathrm{a})}$ \\
\hline \multicolumn{3}{|c|}{ Induced labor group } \\
\hline VD $(n=135)$ & $4(0-8)$ & $17.6 \pm 8.0$ \\
\hline$C D(n=29)$ & $3(0-6)$ & $22.9 \pm 8.3$ \\
\hline$P$-value & $0.002^{a)}$ & $0.002^{\mathrm{a})}$ \\
\hline OR & 1.619 & 0.913 \\
\hline $95 \% \mathrm{Cl}$ & $1.308-2.005$ & $0.872-0.955$ \\
\hline$P$-value & $<0.001^{\text {a) }}$ & $<0.001^{\text {a) }}$ \\
\hline
\end{tabular}

Values are presented as the mean \pm standard deviation or the median (range).

$V D$, vaginal delivery; $C D$, Cesarean delivery; $O R$, odds ratio; $\mathrm{Cl}$, confidence interval.

a) Statistical significance.

group (75.0\% vs. $50.4 \%, P=0.001$ ) (Table 2 ).

Regarding neonatal outcomes, Apgar scores at 1 and 5 minutes were not different between the 2 groups, and the rate of Apgar scores less than 7 at 5 minutes was not different between the groups. The rate of meconium-stained amniotic fluid diagnosed at delivery and the neonatal intubation rate were also not different between the groups. In this 


\section{Obstetrics \& Gynecology Science}

Hye In Kim, et al. Induction, labor, risks

Table 4. Indications for Cesarean delivery

\begin{tabular}{lcc}
\hline Indications & $\begin{array}{c}\text { Spontaneous } \\
\text { labor }(\mathbf{n = 9 )}\end{array}$ & $\begin{array}{c}\text { Induced labor } \\
(\mathbf{n}=\mathbf{2 9})\end{array}$ \\
\hline Fetal distress & $3(33.3)$ & $8(27.6)$ \\
Chorioamnionitis $^{\mathrm{a})}$ & $0(0.0)$ & $1(3.4)$ \\
Failure to progress & $4(44.4)$ & $12(41.4)$ \\
Prolonged 2nd stage & $0(0.0)$ & $5(17.2)$ \\
Unstable lie $^{\text {b) }}$ & $0(0.0)$ & $1(3.4)$ \\
Maternal request during labor & $2(22.2)$ & $2(6.8)$ \\
\hline
\end{tabular}

Values are presented as number (\%).

a) Diagnosed as a Cesarean delivery indication when the maternal body temperature was above $38.3^{\circ} \mathrm{C}$ with maternal tachycardia

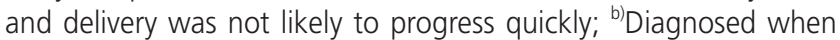
the baby changed its presentation to facial presentation during the labor.

study, the indication for NICU admission included a long duration from admission to delivery in addition to other medical problems. The NICU admission rate was higher in the induction group than in the spontaneous labor group, with statistical significance ( $28.0 \%$ vs. $13.2 \%, P=0.001)$ (Table 2 ).

Our data showed that women with Cesarean delivery had a lower initial Bishop score in both the spontaneous labor and induced labor groups. The Bishop score was higher, and the cervix length was shorter, in women with vaginal delivery than in those with Cesarean delivery. Successful vaginal delivery following induced labor was significantly higher with a shorter cervical length and a higher Bishop score (Table 3).

The reasons for Cesarean delivery are shown in Table 4, and failure to progress was the most common. An unstable lie was diagnosed when the baby changed its vertex presentation to facial presentation.

\section{Discussion}

Many retrospective cohort studies of nulliparous women with vertex, singleton, term pregnancies have reported that the rate of Cesarean delivery is increased approximately 2 -fold in women who undergo induction of labor compared with those who experience spontaneous labor $[8,9,28-31]$. However, the spontaneous labor group in these studies included an expectant management group in the mixture and women who were not induced (i.e., managed expectantly) may have had induction or Cesarean delivery recommended later in pregnancy for a medical indication. Remarkably, when electively induced nulliparous or multiparous women are compared with the appropriate comparison group (i.e., those who are expectantly managed), there is no convincing evidence that elective induction is associated with an increased rate of Cesarean delivery, regardless of whether the cervix is favorable [11-14].

Induced labor can reduce maternal anxiety and discomfort related to normal pregnancy, especially when the patient lives far from the hospital or has a history of previous pregnancy with labor abnormalities, or in case of concern for rapid labor in multiparous women.

In the present study, Cesarean section rates and the length of hospital stay were similar between the 2 groups. It has been stated that postpartum hemorrhage from uterine atony is more common in women undergoing induction or augmentation. Indeed, in one study, labor induction was associated with $17 \%$ of 553 emergency peripartum hysterectomies [32]. In contrast to this previous study, the blood loss during labor (i.e., the decreased hemoglobin level after delivery) was not different between the groups in the current analysis. Among maternal complications, only the delivery time from admission was longer in the induced labor group than in the spontaneous labor group. Regarding neonatal complications, with the exception of the NICU admission rate, other factors (Apgar scores at 1 and 5 minutes, intubation rate, and Apgar score less than 7 at 5 minutes) were similar between the groups. Although the average gestational age between the 2 groups was not different, the number of pregnant women after the estimated delivery date was higher in the spontaneous labor group than in the induced labor group.

The higher NICU admission rate in the induced labor group is thought to be due to early amniotomy as one of the methods of induction. Early amniotomy increased the duration from rupture of membranes to the delivery time. If the time from rupture to delivery is longer than 18 hours, the pediatrics department requires neonates be admitted to the NICU for close observation and the administration of prophylactic antibiotics because of the risk for sepsis, regardless of their condition at delivery. In reality, the Apgar score, which reflects neonatal health status more accurately, was not different between the 2 groups.

This study showed that women who delivered by Cesarean section had unfavorable cervical parameters compared to those who delivered vaginally. With the exception of 1 case, no woman who delivered by Cesarean delivery had a Bishop 


\section{Obstetrics \& Gynecology Science}

Vol. 62, No. 1, 2019

score higher than 6, meaning that a favorable cervix is one of the most important factors for vaginal delivery. However, the overall vaginal delivery rate at term was as high as $84 \%$; thus, we favored induced labor as opposed to elective Cesarean, even when the women had an unfavorable cervix at term. The observed Cesarean section rate of $16 \%$ justifies an attempt at vaginal delivery, as this will also result in fewer complications in both present and future pregnancies. As may be expected, providers need to discuss the delivery mode with women and caregivers. Indeed, Bernardes et al. [13] reported that induction of labor at or near term in women with a median Bishop score of 3 (range, 1-6) was not associated with a higher rate of Cesarean delivery than spontaneous labor, and approximately $85 \%$ of women in both groups achieved vaginal delivery.

Studies of uncomplicated pregnancies reported a longer time to delivery and higher cost associated with labor induction $[33,34]$. The time to delivery was longer for labor induction than for spontaneous labor. However, the time required for cervical ripening in labor induction was not long enough to cause the longer hospital stays. Because waiting for spontaneous labor requires additional antepartum medical visits weekly, labor induction at 39 or more weeks could be less costly.

This study has several strengths. First, we were able to obtain complete records from a single institution with a uniform protocol for analysis, including neonatal Apgar scores. Second, in order to reduce inter-observer variations, pelvic examination and ultrasonography were performed by only 1 expert. Third, unlike previous studies, from the beginning we excluded women who would likely undergo Cesarean delivery from this study, such as those with severe maternal complications or those with fetuses with severe intrauterine growth restriction or large for gestational age. Therefore, we could adjust bias of the Cesarean delivery rate to favor the control group.

However, this study also has several limitations. First, the study included a small number of women, which may limit the ability to generalize our results. Second, we had more women in the induced labor group than in the spontaneous labor group. Most women chose induced labor because of maternal circumstances, as they feared passing their due date.

In conclusion, maternal and neonatal outcomes, including the Cesarean delivery rate, were similar when labor was in- duced at 39 or more weeks of gestation compared to spontaneous labor in uncomplicated, nulliparous women. Our result suggests that induced labor in nulliparous women at full term may be acceptable, even when the indication is only relative for provider and maternal convenience.

Further properly designed, long-term studies on a larger scale are needed to evaluate the precise effects of induced labor on mothers and neonates.

\section{Acknowledgements}

This research was supported by grants from National Health Insurance Service Ilsan Hospital.

\section{Conflict of interest}

No potential conflict of interest relevant to this article was reported.

\section{Ethical approval}

The study was approved by the National Health Insurance Service Ilsan Hospital Institutional Review Board (\#NHIMC 2017-01-047) and performed in accordance with the principles of the Declaration of Helsinki. Informed consent was waived because of the retrospective study design.

\section{Patient consent}

Informed consent was waived because of the retrospective study design.

\section{References}

1. Martin JA, Hamilton BE, Ventura SJ, Osterman MJ, Kirmeyer S, Mathews TJ, et al. Births: final data for 2009. Natl Vital Stat Rep 2011;60:1-70.

2. Calder AA, Loughney AD, Weir CJ, Barber JW. InduCtion of labour in nulliparous and multiparous women: a UK, multicentre, open-label study of intravaginal 


\section{Obstetrics \& Gynecology Science}

Hye In Kim, et al. Induction, labor, risks

misoprostol in comparison with dinoprostone. BJOG 2008;115:1279-88.

3. Clark SL, Miller DD, Belfort MA, Dildy GA, Frye DK, Meyers JA. Neonatal and maternal outcomes associated with elective term delivery. Am J Obstet Gynecol 2009;200:156.e1-4.

4. Ehrenthal DB, Hoffman MK, Jiang X, Ostrum G. Neonatal outcomes after implementation of guidelines limiting elective delivery before 39 weeks of gestation. Obstet Gynecol 2011;118:1047-55.

5. Mishanina E, Rogozinska E, Thatthi T, Uddin-Khan R, Khan KS, Meads C. Use of labour induction and risk of cesarean delivery: a systematic review and meta-analysis. CMAJ 2014;186:665-73.

6. Rosenstein MG, Cheng YW, Snowden JM, Nicholson JM, Caughey AB. Risk of stillbirth and infant death stratified by gestational age. Obstet Gynecol 2012;120:76-82.

7. De los Santos-Garate AM, Villa-Guillen M, VillanuevaGarcía D, Vallejos-Ruíz ML, Murguía-Peniche MT; NEOSANO's Network. Perinatal morbidity and mortality in late-term and post-term pregnancy. NEOSANO perinatal network's experience in Mexico. J Perinatol 2011;31:789-93.

8. Luthy DA, Malmgren JA, Zingheim RW. Cesarean delivery after elective induction in nulliparous women: the physician effect. Am J Obstet Gynecol 2004;191:1511-5.

9. Vahratian A, Zhang J, Troendle JF, Sciscione AC, Hoffman MK. Labor progression and risk of cesarean delivery in electively induced nulliparas. Obstet Gynecol 2005;105:698-704.

10. Bishop EH. Pelvic scoring for elective induction. Obstet Gynecol 1964;24:266-8.

11. Bailit JL, Grobman W, Zhao Y, Wapner RJ, Reddy UM, Varner MW, et al. Nonmedically indicated induction vs expectant treatment in term nulliparous women. Am J Obstet Gynecol 2015;212:103.e1-7.

12. Darney BG, Snowden JM, Cheng YW, Jacob L, Nicholson $J M$, Kaimal A, et al. Elective induction of labor at term compared with expectant management: maternal and neonatal outcomes. Obstet Gynecol 2013;122:761-9.

13. Bernardes TP, Broekhuijsen K, Koopmans CM, Boers KE, van Wyk $L$, Tajik $P$, et al. Caesarean section rates and adverse neonatal outcomes after induction of labour versus expectant management in women with an unripe cervix: a secondary analysis of the HYPITAT and DIGITAT trials. BJOG 2016;123:1501-8.

14. Gülmezoglu AM, Crowther CA, Middleton P, Heatley E. Induction of labour for improving birth outcomes for women at or beyond term. Cochrane Database Syst Rev 2012;(6):CD004945.

15. Saccone G, Berghella V. Induction of labor at full term in uncomplicated singleton gestations: a systematic review and metaanalysis of randomized controlled trials. Am J Obstet Gynecol 2015;213:629-36.

16. Miller NR, Cypher RL, Foglia LM, Pates JA, Nielsen PE. Elective induction of labor compared with expectant management of nulliparous women at 39 weeks of gestation: a randomized controlled trial. Obstet Gynecol Surv 2016;71:197-8.

17. American College of Obstetricians and Gynecologists. ACOG committee opinion no. 561: nonmedically indicated early-term deliveries. Obstet Gynecol 2013;121:911-5.

18. Zhang $X$, Kramer MS. Variations in mortality and morbidity by gestational age among infants born at term. J Pediatr 2009;154:358-62, 362.e1.

19. Wang ML, Dorer DJ, Fleming MP, Catlin EA. Clinical outcomes of near-term infants. Pediatrics 2004;114:372-6.

20. Parikh LI, Reddy UM, Männistö T, Mendola P, Sjaarda L, Hinkle $S$, et al. Neonatal outcomes in early term birth. Am J Obstet Gynecol 2014;211:265.e1-11.

21. Osterman MJ, Martin JA. Recent declines in induction of labor by gestational age. NCHS Data Brief 2014;(155):1-8.

22. Dublin S, Johnson KE, Walker RL, Avalos LA, Andrade SE, Beaton SJ, et al. Trends in elective labor induction for six United States health plans, 2001-2007. J Womens Health (Larchmt) 2014;23:904-11.

23. American College of Obstetrics and Gynecologists. Support for elective IOL at 39 weeks growing [Internet]. Washington, D.C.: American College of Obstetricians and Gynecologists; 2017 [cited 2018]. Available from: http://annualmeeting.acog.org/support-for-elective-iolat-39-weeks-growing.

24. Dietz PM, Rizzo JH, England LJ, Callaghan WM, Vesco KK, Bruce FC, et al. Early term delivery and health care utilization in the first year of life. J Pediatr 2012;161:234-9.e1.

25. Reis FM, Gervasi MT, Florio P, Bracalente G, Fadalti M, Severi FM, et al. Prediction of successful induction of labor at term: role of clinical history, digital examination, 


\section{Obstetrics \& Gynecology Science}

Vol. 62, No. 1, 2019

ultrasound assessment of the cervix, and fetal fibronectin assay. Am J Obstet Gynecol 2003;189:1361-7.

26. Induction and augmentation of labor. In: Cunningham FG, Leveno KJ, Bloom SL, Spong CY, Dashe JS, Hoffman BL et al., editors. Williams obstetrics. 24th ed. New York (NY): McGraw-Hill Education; 2014. p.523-34.

27. Crane JM. Factors predicting labor induction success: a critical analysis. Clin Obstet Gynecol 2006;49:573-84.

28. Cammu H, Martens G, Ruyssinck G, Amy JJ. Outcome after elective labor induction in nulliparous women: a matched cohort study. Am J Obstet Gynecol 2002;186:240-4.

29. Seyb ST, Berka RJ, Socol ML, Dooley SL. Risk of cesarean delivery with elective induction of labor at term in nulliparous women. Obstet Gynecol 1999.94:600-7.

30. Prysak M, Castronova FC. Elective induction versus spontaneous labor: a case-control analysis of safety and efficacy. Obstet Gynecol 1998;92:47-52.

31. Ehrenthal DB, Jiang $X$, Strobino DM. Labor induction and the risk of a Cesareancesarean delivery among nulliparous women at term. Obstet Gynecol 2010;116:3542.

32. Hernandez JS, Wendel GD Jr, Sheffield JS. Trends in emergency peripartum hysterectomy at a single institution: 1988-2009. Am J Perinatol 2013.30:365-70.

33. Maslow AS, Sweeny AL. Elective induction of labor as a risk factor for cesarean delivery among low-risk women at term. Obstet Gynecol 2000;95:917-22.

34. Osmundson S, Ou-Yang RJ, Grobman WA. Elective induction compared with expectant management in nulliparous women with an unfavorable cervix. Obstet Gynecol 2011;117:583-7. 